atoms in crystals can be expressed in terms of atomic radii characteristic only of the element concerned, it is suggested that the particular element is of secondary importance, for elements whose atoms are of the same rare-gas type, and that the contribution of an atom to the distance between it and another atom in a crystal depends upon the number and arrangement of electrons about its positive nucleus, upon the type of lattice, at least as this affects the latter, and, ordinarily, upon the magnitude of the charge carried by the atom.

The inter-atomic distances in substances crystallizing in the diamond type of lattice have been analyzed from this point of view.

PRINCETON, NEW JERSEY

[Contribution from the Gates Chemical Laboratory, California Institutet OF Technology, No. 11]

\title{
THE CRYSTAL STRUCTURES OF COMPLEX CYANIDES OF POTASSIUM WITH ZINC, CADMIUM AND MERCURY
}

By Roscoe G. DickINSON 1

Received February 13, 1922

\section{Introduction}

The complex cyanides $\mathrm{K}_{2} \mathrm{Zn}(\mathrm{CN})_{4}, \mathrm{~K}_{2} \mathrm{Cd}(\mathrm{CN})_{4}, \mathrm{~K}_{2} \mathrm{Hg}(\mathrm{CN})_{4}, \mathrm{Tl}_{2} \mathrm{Zn}(\mathrm{CN})_{4}$, and $\mathrm{T}_{2} \mathrm{Hg}(\mathrm{CN})_{4}$ are mentioned by Groth ${ }^{2}$ as probably crystallizing with cubic symmetry; but apparently only the first compound has been shown to be optically isotropic. In the work to be here described crystals of the first three of these compounds were prepared and found to be optically isotropic. Their structures were also determined by Xray methods. The relation of the new structures to those of some other crystals, as well as the magnitude of the interatomic distances, present some interesting features which will be pointed out in the latter part of this article.

\section{Materials and Methods of Experimentation}

Potassium zinc cyanide was prepared by adding a solution of potassium cyanide (imported "sodium free" potassium cyanide was used throughout) to zinc sulfate solution, washing the precipitate, and dissolving it in warm, conc. potassium cyanide solution, from which the product separated on cooling.

Potassium cadmium cyanide was prepared by adding ammonium hydroxide to cadmium nitrate solution, washing the precipitate, and dissolving it in potassium cyanide solution.

Potassium mercury cyanide was obtained by adding mercuric oxide to potassium cyanide solution, and recrystallizing the product.

In all cases the crystals used were obtained by slow evaporation of their aqueous

${ }^{2}$ National Research Fellow in Chemistry.

2 Groth, "Chemische Krystallographie," Engelmann, Leipzig, 1906. Vol. 1, p. $334, \mathrm{ff}$. 
solutions at room temperature; this method readily gave crystals $1 \mathrm{sq} . \mathrm{cm}$. or more in cross section. They were octahedral in habit. No definite cleavage was observed.

The densities of the crystals were determined on small specimens by the Retgers suspension method. They were found to be: $\mathrm{K}_{2} \mathrm{Zn}(\mathrm{CN})_{4}, 1.673 ; \mathrm{K}_{2} \mathrm{Cd}(\mathrm{CN})_{4}, 1.847$; $\mathrm{K}_{2} \mathrm{Hg}(\mathrm{CN})_{4}, 2.434$.

The X-ray data were obtained in two ways: (1) from spectrographic measurements of the characteristic rays from a rhodium target, using calcite as a reference crystal; (2) from unsymmetrical Laue photographs. As the apparatus and routine parts of the treatment of the data were substantially the same as those previously used, ${ }^{3}$ no further description of the apparatus or restatement of formulas used will be given.

\section{Spectral Photographic Data}

In Tables I, II and III are given the angles of reflection of the rhodium lines $\beta=0.5453 \AA$. and $\alpha=0.614 \AA$. (the doublet being not completely resolved), and the estimated intensities of the reflections.

To get the number of molecules $m$ in the unit of structure, the strongest reflection from the (111) face may conveniently be used. If $n$ is the order of reflection, the values of $n^{3} / m$ are found in the usual way to be: $\mathrm{K}_{2} \mathrm{Zn}(\mathrm{CN})_{4}, 3.355 ; \mathrm{K}_{2} \mathrm{Cd}(\mathrm{CN})_{4}, 3.35 ; \mathrm{K}_{2} \mathrm{Hg}(\mathrm{CN})_{4}, 3.365$. From this it follows that $m=8$, and for this reflection $n=3$, which gives $n^{3} / m=3.365$. The order of each other reflection may readily be shown to have the value given in the fourth column of the tables. The value of $d_{100}$, the length of the unit of structure, calculated from each reflection, is given in the fifth column.

TABLE I

\begin{tabular}{|c|c|c|c|c|c|c|}
\hline & & LECTION D & 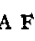 & POTAS & INNC CyaNtDE & \\
\hline Plane & Line & $\begin{array}{l}\text { Observed angle } \\
\text { of refection }\end{array}$ & $n$ & $d_{100}$ & Intensity & $\sqrt{A^{2}+B^{2}}$ \\
\hline (111) & $\alpha$ & $2^{\circ} 27^{\prime}$ & 1 & $(12.4)$ & faint & 35 \\
\hline & & & 2 & & absent & 30 \\
\hline & $\beta$ & $6^{\circ} 29^{\prime}$ & 3 & 12.54 & & \\
\hline & $\alpha$ & $7^{\circ} 19^{\prime}$ & 3 & 12.52 & medium & 342 \\
\hline & $\alpha$ & $9^{\circ} 47^{\prime}$ & 4 & 12.52 & very weak & 229 \\
\hline & $\alpha$ & $12^{\circ} 16^{\prime}$ & 5 & 12.51 & weak & 334 \\
\hline$(100)$ & $\beta$ & $4^{\circ} 59^{\prime}$ & 4 & 12.55 & & \\
\hline & $\alpha$ & $5^{\circ} 37^{\prime}$ & 4 & 12.55 & medium & 365 \\
\hline & $\beta$ & $10^{\circ} \quad 3^{\prime}$ & 8 & 12.50 & & \\
\hline & $\alpha$ & $11^{\circ} 18^{\prime}$ & 8 & 12.53 & medium & 569 \\
\hline (110) & $\beta$ & $3^{\circ} 31^{\prime}$ & 2 & 12.57 & & \\
\hline & $\alpha$ & $3^{\circ} 58^{\prime}$ & 2 & 12.55 & medium strong & 297 \\
\hline & $\beta$ & $7^{\circ} \quad 1^{\prime}$ & 4 & 12.61 & & \\
\hline & $\alpha$ & $7^{\circ} 57^{\prime}$ & 4 & 12.56 & very strong & 764 \\
\hline & $\beta$ & $10^{\circ} 37^{1 / 2^{\prime}}$ & 8 & 12.54 & & \\
\hline & $\alpha$ & $11^{\circ} 59^{\prime}$ & 8 & 12.56 & medium & 572 \\
\hline & & Calc. from & $\alpha$. & $\begin{array}{l}12.54 \\
12.50\end{array}$ & & \\
\hline
\end{tabular}

Dickinson, This Journal, 44, 276 (1922). 
TABLE II

\begin{tabular}{|c|c|c|c|c|c|c|}
\hline Plane & $\begin{array}{l}\text { REFL, } \\
\text { Line }\end{array}$ & $\begin{array}{l}\text { CTION DATA I } \\
\text { Observed angle } \\
\text { of reflection }\end{array}$ & $\mathrm{OR} P$ & $\begin{array}{l}\text { ASSIUM CA } \\
d_{i 00}\end{array}$ & $\begin{array}{l}\text { IIUM CYA } \\
\text { Intensity }\end{array}$ & $\sqrt{4^{2}+B^{2}}$ \\
\hline \multirow[t]{6}{*}{ (111) } & $\alpha$ & $2^{\circ} 21^{\prime}$ & $\begin{array}{l}1 \\
2\end{array}$ & $(13.0-)$ & $\begin{array}{l}\text { weak } \\
\text { absent }\end{array}$ & $\begin{array}{r}137 \\
30\end{array}$ \\
\hline & $\beta$ & $6^{\circ} 20^{\prime}$ & 3 & 12.84 & & \\
\hline & $\alpha$ & $7^{\circ} \quad 8^{\prime}$ & 3 & 12.85 & medium & 444 \\
\hline & & & 4 & & absent & 85 \\
\hline & $\alpha$ & $11^{\circ} 57^{\prime}$ & 5 & 12.84 & weak & 436 \\
\hline & & Calc. fro & $\begin{array}{l}\text { Mean } \\
\text { densi }\end{array}$ & $\begin{array}{l}12.84 \\
12.81 \AA\end{array}$ & & \\
\hline
\end{tabular}

TABLE III

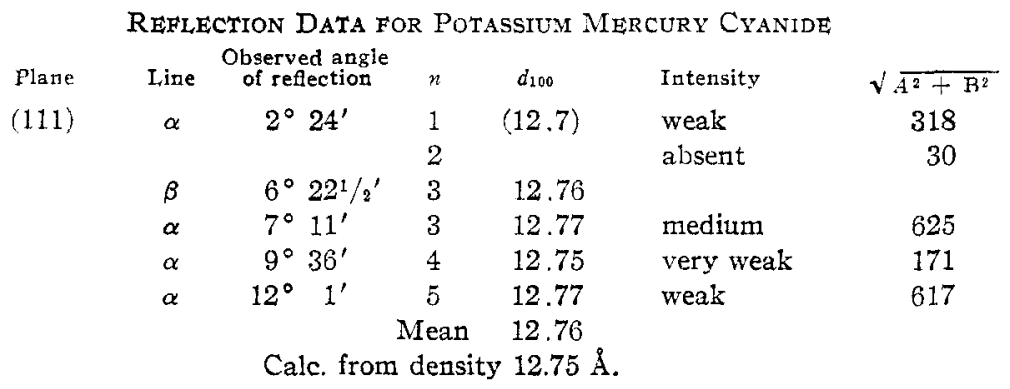

\section{Application of the Theory of Space Groups}

The simple requirement that there shall be 8 molecules in the unit still leaves as possible a considerable variety of structures. The additional assumption, however, that all the atoms of each element are situated in equivalent positions in the structure limits the possibilities to those space groups which can give $8,16,32$, and 32 equivalent positions. These space groups are: ${ }^{4} \mathrm{~T}_{\mathrm{h}}{ }^{4}, \mathrm{O}^{4}, \mathrm{O}_{\mathrm{h}}{ }^{7}$. Each of these leads to the same possible arrangements of atoms; and it will be shown that a structure can be derived from them that is in satisfactory accord with the experimental data.

The possible positions for the atoms are:

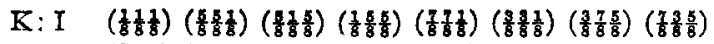

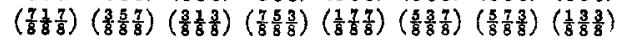

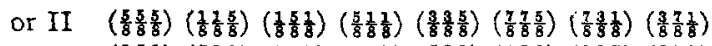

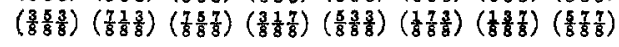

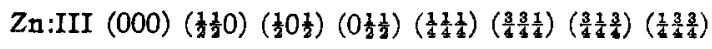

$$
\begin{aligned}
& \text { or } \operatorname{IV}\left(\frac{1}{2} \frac{1}{2} \frac{1}{2}\right)\left(00 \frac{1}{2}\right)\left(0 \frac{1}{2} 0\right)\left(\frac{1}{2} 00\right)\left(\frac{3}{4} \frac{3}{4}\right)\left(\frac{1}{4} \frac{1}{4} \frac{3}{4}\right)\left(\frac{1}{4} \frac{3}{4} \frac{1}{4}\right)\left(\frac{3}{4} \frac{1}{4} \frac{1}{4}\right)
\end{aligned}
$$

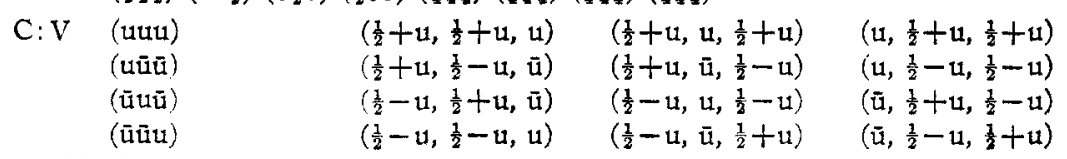

"Niggli, "Geometrische Krystallographie des Diskontinuums," Bornträger, Leipzig, 1919, p. 410; also Phys. Zeit., 19, 229 (1918). 
$\left(\frac{1}{4}-u, \frac{1}{4}-u, \frac{1}{4}-u\right)\left(\frac{3}{4}-u, \frac{3}{4}-u, \frac{1}{4}-u\right)\left(\frac{3}{4}-u, \frac{1}{4}-u, \frac{3}{4}-u\right)\left(\frac{1}{4}-u, \frac{3}{4}-u, \frac{3}{4}-u\right)$
$\left(\frac{1}{4}-u, \frac{1}{4}+u, \frac{1}{4}+u\right)\left(\frac{3}{4}-u, \frac{3}{4}+u, \frac{1}{4}+u\right)\left(\frac{3}{4}-u, \frac{1}{4}+u, \frac{3}{4}+u\right)\left(\frac{1}{4}-u, \frac{3}{4}+u, \frac{3}{4}+u\right)$
$\left(\frac{1}{4}+u, \frac{1}{4}-u, \frac{1}{4}+u\right)\left(\frac{3}{4}+u, \frac{3}{4}-u, \frac{1}{4}+u\right)\left(\frac{3}{4}+u, \frac{1}{4}-u, \frac{3}{4}+u\right)\left(\frac{1}{4}+u, \frac{3}{4}-u, \frac{3}{4}+u\right)$
$\left(\frac{1}{4}+u, \frac{1}{4}+u, \frac{1}{4}-u\right)\left(\frac{3}{4}+u, \frac{3}{4}+u, \frac{1}{4}-u\right)\left(\frac{3}{4}+u, \frac{1}{4}+u, \frac{3}{4}-u\right)\left(\frac{1}{4}+u, \frac{3}{4}+u, \frac{3}{4}-u\right)$

$\mathrm{N}$ : VI A similar set of coördinates in $\mathrm{v}$.

It is to be noted that all distinct combinations of the above coördinates are obtained by combining V, VI, and either I or II with III; and by giving $\mathrm{u}$ and $\mathrm{v}$ any independent values between $1 / 8$ and $5 / 8$. Either of these combinations can be shown to have the following properties: for the plane (100) all reflections except the 4 th, 8 th.... orders must be absent; for the plane (110), all odd orders must be absent; for (111) any order may be present. This agrees with the observations.

A treatment of the possibilities arising from complete independence of $\mathrm{u}$ and $\mathrm{v}$ is laborious, and from a practical point of view unnecessary, since the carbon and nitrogen atoms may safely be assumed to be closely associated. Thus the sum of the radii assigned by Bragg ${ }^{5}$ to carbon and nitrogen is $1.42 \AA$; ; and Bozorth ${ }^{6}$ obtained best agreement in the case of potassium cyanide by placing the carbon and nitrogen $1.15 \AA$. apart. It therefore seems safe to assume that the distance of the carbon from the nitrogen lies between 1 and $1.5 \AA$., which is equivalent to assuming that

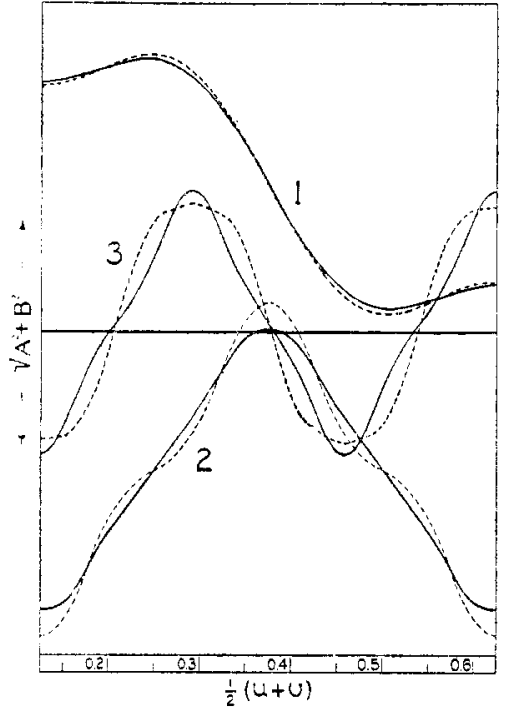

Fig. 1.-Values $\sqrt{A^{2}+B^{2}}$ for the first three orders from $\mathrm{K}_{2} \mathrm{Zn}(\mathrm{CN})_{4}$, (11I); the abscissas are $\frac{1}{2}(u+v)$. The potassium atoms have Arrangement $I$.

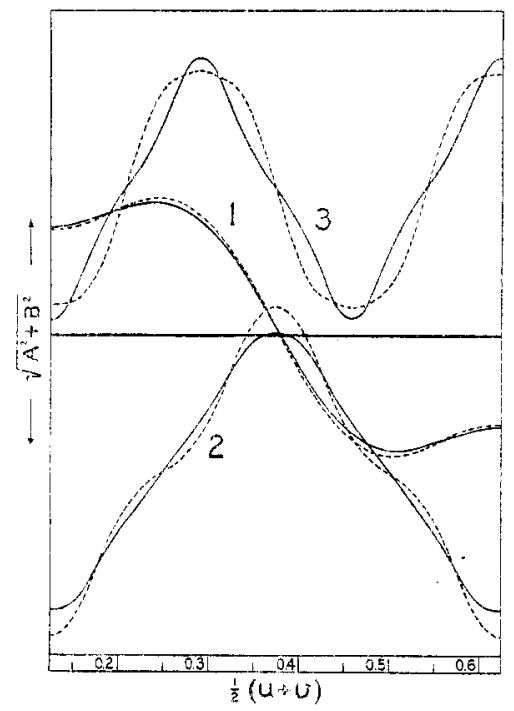

Fig. 2.-Values of $\sqrt{A^{2}+\bar{B}^{2}}$ for the first three orders from $\mathrm{K}_{2} \mathrm{Zn}(\mathrm{CN})_{4}$, (111); the abscissas are $\frac{1}{2}(u+v)$. The potassium atoms have Arrangement II.

s Bragg, Phil. Mag., 40, 180 (1920).

${ }^{6}$ Bozorth, This Journal, 44, 317 (1922). 
in the zinc compound, $\mathrm{u}$ differs from $\mathrm{v}$ by not less than 0.046 and not more than 0.069 .

The intensities of the first three orders of reflection from (111) afford a means of discriminating between the two possible locations of the potassium atoms, and of locating the cyanide group approximately without employing any quantitative assumption of the "normal decline" of intensity. In Figs. 1 and 2 are shown the values of $\sqrt{\mathrm{A}^{2}+\mathrm{B}^{2}}$ for the (111) reflections from $\mathrm{K}_{2} \mathrm{Zn}(\mathrm{CN})_{4}$ calculated in the usual manner. The reflecting powers of the atoms have been taken proportional to the atomic numbers, except for carbon and nitrogen, whose reflecting powers have each been taken proportional to 6.5 , instead of to 6 and 7 , respectively, since it does not seem possible to distinguish these two atoms. In Fig. 1 the potassium atoms have the arrangement I, and in Fig. 2 the arrangement II. The abscissas, $1 / 2(u+v)$, are positions of a point half-way between the centers of the carbon and nitrogen atoms. The solid curves are for a distance of $1.5 \AA$. between carbon and nitrogen, and the dotted curves for a distance of $1.0 \AA$. The only positions where the second order has a small ordinate while the third is much larger than the first, as the observed intensities evidently require, are in Fig. 2 in the neighborhood of $1 / 2(u+v)=0.375$. It is concluded, then, that the potassium atoms have the positions given by II and that $1 / 2(u+v)$ is about $3 / 8$. This structure is capable of accounting for the other abnormalities of the spectra in the same manner. In the last column of Tables I, II and III are given the values of $\sqrt{A^{2}+B^{2}}$ calculated by placing $\mathrm{u}=0.34$ and $\mathrm{v}=$ 0.40 with the potassium atoms in Position II. On any face where stronger reflections occur at larger angles, the value of the factor $\sqrt{A^{2}+B^{2}}$ is greater; and where the reflections are weak at small angles, the value of the factor is small.

\section{The Laue Photographic Data}

Unsymmetrical Laue photographs were made with $\mathrm{K}_{2} \mathrm{Zn}(\mathrm{CN})_{4}$, using slips of crystal approximately parallel to (100) and to (111); while with $\mathrm{K}_{2} \mathrm{Cd}(\mathrm{CN})_{4}$ and $\mathrm{K}_{2} \mathrm{Hg}(\mathrm{CN})_{4}$ the $\mathrm{X}$-ray beam was only passed approximately perpendicular to (111). As over a thousand spots were examined, a tabulation of the complete data is impracticable. A number of useful and representative spots have been chosen from each of three photographs; and the indices, estimated intensities, spacings of planes, and values of $n \lambda$ for these are given in Tables IV, V and VI. The nature of the change of intensity with wavelength is shown by the inclusion of several planes of one form.

The expressions for $\mathrm{A}$ and $\mathrm{B}$ for cases which will be considered can be simplified to the following. 
A. Two indices even.

For $n=1, A=0$, and $B=0$.

For $n=2, A=0$, and $B=8 \Sigma\left(\overline{\mathrm{C}} \sin 4 \pi S_{n} \mathrm{u}+\overline{\mathrm{N}} \sin 4 \pi S_{n} \mathrm{v}\right)$

B. One index even.

For $n=1, A=0$, and $B=0$.

For $n=2, A=8 \overline{\mathrm{Zn}}+8 \Sigma\left(\overline{\mathrm{C}} \cos 4 \pi S_{n} \mathrm{u}+\overline{\mathrm{N}} \cos 4 \pi S_{n} \mathrm{v}\right)$ and $B=0$.

C. All indices odd, and $n=1$.

$$
\begin{aligned}
& A=4 \overline{\mathrm{Zn}}+4 \Sigma\left[ \pm \overline{\mathrm{K}} \cos \frac{\pi S_{n}}{4}+\overline{\mathrm{C}}\left(\cos 2 \pi S_{n} \mathrm{u}+\cos 2 \pi\left(\frac{S_{1}}{4}-S_{n} \mathrm{u}\right)\right)\right. \\
& \left.+\overline{\mathrm{N}}\left(\cos 2 \pi S_{n} \mathrm{v}+\cos 2 \pi\left(\frac{S_{1}}{4}-S_{n} \mathrm{v}\right)\right)\right] \\
& B=4 \Sigma\left[\overline{Z n} \sin \frac{\pi S_{n}}{2} \pm \overline{\mathrm{K}} \sin \frac{\pi S_{n}}{4}+\bar{C}\left(\sin 2 \pi S_{n} \mathrm{u}+\sin 2 \pi\left(\frac{S_{1}}{4}-S_{n} \mathrm{u}\right)\right)\right. \\
& \left.+\mathrm{N}\left(\sin 2 \pi S_{n} \mathrm{v}+\sin 2 \pi\left(\frac{S_{1}}{4}-S_{n} \mathrm{v}\right)\right)\right]
\end{aligned}
$$

where $\mathrm{h}+\mathrm{k}+1=S_{1} ; \mathrm{h}-\mathrm{k}-1=S_{2} ;-\mathrm{h}+\mathrm{k}-1=S_{3} ;-\mathrm{h}-\mathrm{k}+$ $1=S_{4}$. The summations are in all cases to be taken from $n=1$, to $n$ $=4$. The sign before the $\overline{\mathrm{K}}$ term is plus if the potassium atoms have Arrangement I, and minus if they have Arrangement II.

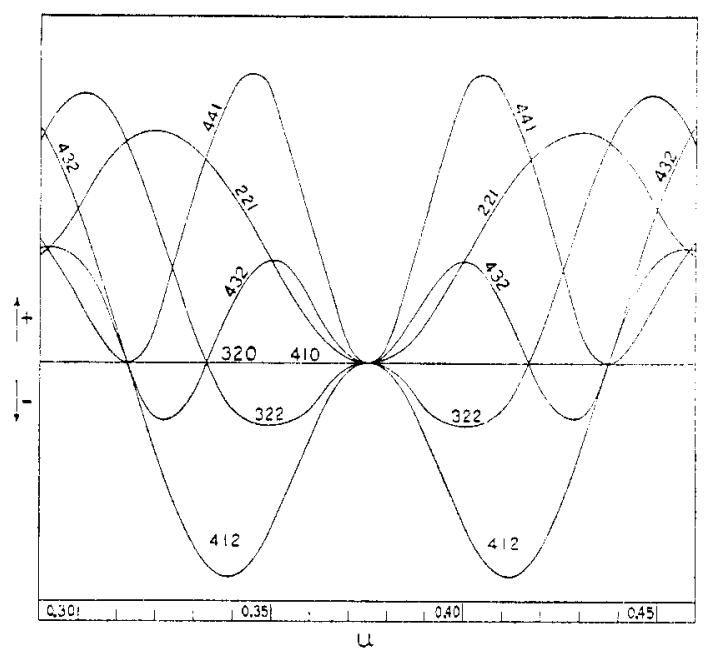

Fig. 3.-Values of $\Sigma \sin 4 \pi \mathrm{S}_{n} \mathrm{u}$ for various planes of Class A.

These equations show that planes having any even index may in general reflect in the second, but not in the first order, and that planes having all odd indices may reflect in the first order. The observations are in agreement with these requirements.

Second order reflections from planes of Class A should be due to the cyanide group alone. Planes of this class having one index zero should not 
appear in the second order for any values of $u$ and $v$; and in fact on the photographs none were found, although some were shown to be favorably situated for reflection. The spots of Class $A$ which did appear in the second order are particularly useful in confirming the location of the cyanide group. A consideration of the data of Tables IV, V and VI and of other similar data shows that for all three salts the following relations must be true of the values of $\sqrt{A^{2}+B^{2}}:$ (441) must be greater than (322) and (432); (421) must be much greater than (322); (221) must be appreciable. The values of $\Sigma \sin 4 \pi S_{n}$ u for these planes are shown plotted against values of $\boldsymbol{u}$ in Fig. 3. The functions defining these curves have the property that if two atoms of equal reflecting power are placed at two different values of the parameter, the resulting value of $\sqrt{A^{2}+B^{2}}$ will be given by the algebraic sum of the corresponding ordinates. The conditions given above are well satisfied by placing one of the two atoms at 0.34 and the other at 0.40 as in the previous calculations; but the presence of two parameters makes a close determination of their values impossible without resort to rather doubtful quantitative assumptions.

The planes having all odd indices may be further divided into two classes. Class $C_{a}$ comprises those which have either only one or all three indices equal to $8 p=3$ (where $p$ is an integer which need not be the same for each index); and class $C_{b}$, those which have either only one or all three indices equal to $8 p \pm 1$. Examples of $C_{a}$ are $(1 \overline{5} 7)$, $(3 \overline{5} 5)$, (95) 1$)$; examples of $\mathrm{C}_{\mathrm{b}}$ are: $(5 \overline{1} 3),(5 \overline{7} 3),(\overline{7} \overline{1})$. When planes of about the same spacing were compared, it was found that those of Class $\mathrm{C}_{\mathrm{a}}$ were distinctly more intense than those of $\mathrm{C}_{\mathrm{b}}$; the intensity difference was greatest with the zinc salt and least with the mercury salt. Neglecting for the moment the cyanide group, the expression for $\sqrt{A^{2}+B^{2}}$ reduces to $4 \sqrt{2 \mathrm{Zn}}-8 \overline{\mathrm{K}}$ for Class $\mathrm{C}_{\mathrm{a}}$ and to $4 \sqrt{2 \mathrm{Zn}}+8 \overline{\mathrm{K}}$ for Class $\mathrm{C}_{\mathrm{b}}$ if the potassium atoms have Arrangement $\mathrm{I}$, and vice versa if they have Arrangement II. There is thus a wide range of new evidence for Arrangement II. This conclusion is not invalidated by the neglect of the cyanide group; for if $\mathrm{v}-3 / 8=3 / 8-\mathrm{u}$ and the carbon and nitrogen reflect equally the $\overline{\mathrm{C}}$ and $\overline{\mathrm{N}}$ terms become zero. The fact that abnormalities in the intensity relations among the planes of class $C_{a}$ alone or $C_{b}$ alone were certainly small is taken to indicate that $\mathrm{u}$ and $\mathrm{v}$ have approximately such values; this test is especially sensitive in the case of Class $C_{b}$ of the zinc compound where the resultant of the zinc and potassium reflections is small.

By careful direct comparison of planes of the same class the following intensity differences in the case of the zinc salt were shown: (951) $>(773)$, $(975)>(11.5 .3),(553)>(731),(771)>(753)$. The differences were small with the zinc salt and very uncertain with the others. The parameters $\mathrm{u}=0.34$ and $\mathrm{v}=0.40$ account for these. 
Planes of Class $B$ also showed very slight abnormalities. With the zinc salt it seemed clear that $\sqrt{A^{2}+B^{2}}$ should stand in the order (501)

TABLE IV

LAUE PhOTOGRAPHic Data from $\mathrm{K}_{2} \mathrm{Zn}(\mathrm{CN})$,

\begin{tabular}{|c|c|c|c|c|c|c|c|}
\hline Plane & $\begin{array}{l}\text { Estimated } \\
\text { intensity }\end{array}$ & Spacing & $n \lambda$ & Plane & $\begin{array}{l}\text { Estimated } \\
\text { intensity }\end{array}$ & Spacing & $n \lambda$ \\
\hline$(1 \overline{2} 2)$ & 1,8 & 4.18 & 0.88 & $(7 \overline{5} 3)$ & 0.05 & 1.38 & 0.55 \\
\hline$(0 \overline{2} 3)$ & 0.0 & 3.48 & 0.82 & $(9 \overline{13})$ & 1.6 & 1.32 & 0.55 \\
\hline$(22 \overline{3})$ & 0.1 & 3.04 & 0.84 & $(\overline{7} 71)$ & 0.4 & 1.26 & 0.46 \\
\hline$(\overline{214})$ & 0.5 & 2.74 & 0.80 & $(9 \overline{5} 1)$ & 1.2 & 1.21 & 0.38 \\
\hline$(\overline{12} 4)$ & 0.1 & 2.74 & 0.60 & $(91 \overline{5})$ & 1.4 & 1.21 & 0.50 \\
\hline (432) & 0.05 & 2.33 & 0.95 & $(37 \overline{7})$ & 1.3 & 1.21 & 0.48 \\
\hline (441) & 0.3 & 2.18 & 0.96 & $(9 \overline{7} 5)$ & 0.8 & 1.01 & 0.42 \\
\hline$(\overline{4} 14)$ & 0.2 & 2.18 & 0.80 & $(5 \overline{\overline{7}} 9)$ & 0.9 & 1.01 & 0.48 \\
\hline$(13 \overline{3})$ & 0.8 & 2.88 & 0.52 & $(\overline{3} \cdot 11 \cdot \overline{5})$ & 0.7 & 1.01 & 0.48 \\
\hline$(31 \overline{3})$ & 1.4 & 2.88 & 0.98 & $(1 \cdot 11 \cdot \overline{7})$ & 0.1 & 0.96 & 0.55 \\
\hline 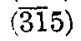 & 0.4 & 2.12 & 0.55 & $(11 \cdot 3 \cdot \overline{7})$ & 0.05 & 0.94 & 0.46 \\
\hline$(3 \overline{5} 5)$ & 6. & 1.63 & 0.44 & $(59 \overline{9})$ & 0.2 & 0.92 & 0.43 \\
\hline$(7 \overline{31})$ & 2. & 1.63 & 0.36 & $(4 \overline{11})$ & 3. & 2.96 & 0.98 \\
\hline$(7 \overline{1} \overline{3})$ & 5. & 1.63 & 0.43 & $(2 \overline{3} 3)$ & 0.8 & 2.67 & 0.81 \\
\hline$(\overline{137})$ & 4. & 1.63 & 0.67 & $(1 \overline{3} 4)$ & 0.9 & 2.46 & 0.77 \\
\hline$(\overline{317})$ & 1.4 & 1.63 & 0.81 & $(41 \overline{3})$ & 1.2 & 2.46 & 0.84 \\
\hline$(71 \overline{3})$ & 0.3 & 1.63 & 0.99 & $(\overline{125})$ & 0.7 & 2.29 & 0.89 \\
\hline$(\overline{3} 7 \overline{3})$ & 0.4 & 1.53 & 0.54 & $(\overline{4} 15)$ & $0.4-$ & 1.94 & 0.95 \\
\hline (157) & 3.5 & 1.45 & 0.40 & $(\overline{1} 6 \overline{3})$ & $0.2-$ & 1.85 & 0.96 \\
\hline$(\overline{5} 7 \overline{1})$ & 3. & 1.45 & 0.55 & $(\overline{5} 34)$ & $0.2-$ & 1.77 & 0.94 \\
\hline$(\overline{7} 35)$ & $0.1+$ & 1.38 & 0.44 & $(0 \overline{3} 7)$ & $0.1+$ & 1.65 & 0.85 \\
\hline
\end{tabular}

TABLE V

LaUe Photographic Data From $\mathrm{K}_{2} \mathrm{Cd}(\mathrm{CN})_{4}$

$\begin{array}{llcc}\text { Plane } & \begin{array}{c}\text { Estimated } \\ \text { intensity }\end{array} & \text { Spacing } & n \lambda \\ (\overline{2} 21) & 0.6 & 4.28 & 0.87 \\ (03 \overline{2}) & 0.0 & 3.56 & 0.92 \\ (22 \overline{3}) & 0.2 & 3.11 & 1.04 \\ (\overline{12} 4) & 0.1 & 2.80 & 0.64 \\ (4 \overline{12}) & 0.4 & 2.80 & 1.20 \\ (3 \overline{4} 2) & 0.0 & 2.38 & 0.86 \\ (4 \overline{4} 1) & 0.1+ & 2.23 & 0.84 \\ (\overline{135}) & 0.8 & 2.17 & 0.40 \\ (\overline{513}) & 1.6 & 2.17 & 0.79 \\ (33 \overline{5}) & 9 . & 1.96 & 0.45 \\ (1 \overline{5} 5) & 0.7 & 1.80 & 0.41 \\ (\overline{5} 53) & 4 . & 1.67 & 0.45 \\ (53 \overline{5}) & 2 . & 1.67 & 0.90 \\ (\overline{5} 71) & 1.0 & 1.48 & 0.34 \\ (7 \overline{15}) & 1.8 & 1.48 & 0.43 \\ (\overline{7} \overline{5}) & 6 . & 1.48 & 0.48 \\ (1 \overline{5}) & 5 . & 1.48 & 0.53 \\ (7 \overline{5} 1) & 1.5 & 1.48 & 0.86 \\ (\overline{5} \overline{7} 3) & 0.2+ & 1.41 & 0.39 \\ (\overline{7} \overline{7} 1) & 0.2 & 1.29 & 0.38\end{array}$

Estimated

Plane intensity Spacing $n \lambda$

$\begin{array}{llll}(\overline{15} 9) & 0.7 & 1.24 & 0.41\end{array}$

$\begin{array}{llll}(915) & 0.4 & 1.24 & 0.87\end{array}$

$\begin{array}{llll}(37 \overline{7}) & 0.6 & 1.24 & 0.42\end{array}$

$\begin{array}{llll}(\overline{7} 57) & 0.5 & 1.16 & 0.41\end{array}$

$\begin{array}{llll}(\overline{3} \cdot 11 \cdot \overline{3}) & 0.3 & 1.09 & 0.40\end{array}$

$(\overline{1} \cdot \overline{5} \cdot 11) \quad 0.1 \quad 1.06 \quad 0.48$

$\begin{array}{llll}(11 \cdot \overline{5} \cdot \overline{3}) & 0.6 & 1.03 & 0.47\end{array}$

$\begin{array}{llll}(5 \overline{9} 7) & 0.3 & 1.03 & 0.40\end{array}$

$\begin{array}{llll}(1 \cdot 11 \cdot \overline{7}) & 0.1 & 0.98 & 0.38\end{array}$

$\begin{array}{llll}(11 \cdot \overline{9} \cdot 1) & 0.1 & 0.90 & 0.38\end{array}$

$\begin{array}{llll}(\overline{5} \cdot \overline{1} \cdot 15) & 0.1 & 0.81 & 0.47\end{array}$

$\begin{array}{llll}(\overline{3} 32) & 0.7 & 2.74 & 0.86\end{array}$

$\begin{array}{llll}(\overline{3} 14) & 0.7 & 2.52 & 0.78\end{array}$

$\begin{array}{llll}\text { (215) } & 0.4 & 2.34 & 0.81\end{array}$

$\begin{array}{llll}(\overline{12} 5) & 0.6 & 2.34 & 0.92\end{array}$

(053) $\quad 0.2-\quad 2.20 \quad 0.72$

$\begin{array}{llll}(0 \overline{3} 5) & 0.2 & 2.20 & 0.92\end{array}$

$\begin{array}{llll}(1 \overline{4} 5) & 0.2 & 1.98 & 0.82\end{array}$

$\begin{array}{llll}(4 \overline{5} 3) & 0.1 & 1.82 & 0.85\end{array}$

$\begin{array}{llll}(\overline{3} 70) & 0.1 & 1.69 & 0.76\end{array}$ 
TABLE VI

\begin{tabular}{|c|c|c|c|c|c|c|c|}
\hline & & LAUE $\mathrm{I}$ & GRA & $\begin{array}{l}\text { LE VI } \\
\text { DATA FRON }\end{array}$ & $\mathrm{K}_{2} \mathrm{Hg}(\mathrm{Cl}$ & & \\
\hline Plane & $\begin{array}{l}\text { Estimated } \\
\text { intensity }\end{array}$ & Spacing & $n \lambda$ & Plane & $\begin{array}{l}\text { Estimated } \\
\text { intensity }\end{array}$ & Spacing & $n \lambda$ \\
\hline$(\overline{2} 21)$ & 0.6 & 4.25 & 1.24 & $(91 \overline{7})$ & 0.6 & 1.11 & 0.35 \\
\hline$(03 \overline{2})$ & 0.0 & 3.54 & 0.74 & $(\overline{5} \cdot 11 \cdot \overline{1})$ & 0.4 & 1.05 & 0.37 \\
\hline$(22 \overline{3})$ & 0.0 & 3.10 & 0.69 & $(\overline{3} \cdot \overline{5} \cdot 11)$ & 0.8 & 1.02 & 0.36 \\
\hline$(\overline{2} \overline{1} 4)$ & 0.3 & 2.78 & 0.79 & $(77 \overline{9})$ & 0.2 & 0.95 & 0.34 \\
\hline$(\overline{12} 4)$ & 0.3 & 2.78 & 0.90 & $(\overline{9} 59)$ & 0.3 & 0.93 & 0.35 \\
\hline$(3 \overline{4} 2)$ & 0.0 & 2.37 & 0.80 & $(\overline{3} \cdot 13 \cdot \overline{3})$ & 0.4 & 0.93 & 0.44 \\
\hline$(4 \overline{4} 1)$ & 0.05 & 2.22 & 0.71 & $(\overline{1} \cdot 13 \cdot \overline{5})$ & 0.2 & 0.91 & 0.42 \\
\hline$(\overline{315})$ & 7. & 2.16 & 0.48 & $(13 \cdot \overline{5} \cdot \overline{3})$ & 0.3 & 0.90 & 0.43 \\
\hline$(1 \overline{5} 5)$ & 3. & 1.79 & 0.48 & $(\overline{3} 32)$ & 1.2 & 2.72 & 1.10 \\
\hline$(7 \overline{33})$ & 3. & 1.56 & 0.33 & $(14 \overline{3})$ & 3.5 & 2.50 & 0.89 \\
\hline (157) & 4. & 1.47 & 0.33 & $(41 \overline{3})$ & 0.9 & 2.50 & 1.13 \\
\hline$(7 \overline{51})$ & 5. & 1.47 & 0.35 & $(\overline{1} 5 \overline{2})$ & 2.5 & 2.33 & 0.69 \\
\hline$(17 \overline{5})$ & 6. & 1.47 & 0.43 & $(\overline{21} 5)$ & 0.7 & 2.33 & 1.07 \\
\hline (517) & 1.2 & 1.47 & 0.57 & $(\overline{4} 33)$ & 1.5 & 2.19 & 0.70 \\
\hline$(71 \overline{5})$ & 1.0 & 1.47 & 0.60 & $(\overline{3} 05)$ & 1.3 & 2.19 & 0.88 \\
\hline$(3 \overline{7} \overline{0})$ & 1.6 & 1.40 & 0.35 & $(51 \overline{4})$ & 0.9 & 1.97 & 0.71 \\
\hline$(\overline{7} 55)$ & 1.1 & 1.28 & 0.35 & $(\overline{2} 7 \overline{1})$ & 0.3 & 1.73 & 0.88 \\
\hline$(519)$ & 2.0 & 1.23 & 0.44 & $(07 \overline{3})$ & 0.3 & 1.68 & 0.83 \\
\hline$(\overline{7} 37)$ & 1.8 & 1.23 & 0.36 & & & & \\
\hline
\end{tabular}

$>(431)>(332)$ for these three planes. The parameters cited account for this order but would lead one to expect larger intensity differences than were observed. If it is attempted to improve the agreement by taking other values of $u$ and $v(e . g .$, by placing the carbon and nitrogen atoms much closer together near 0.375 ) the qualitative account of the spectrographic intensities fails. The difficulty is of the same character as that found by Bozorth with potassium cyanide and is removed in both cases if atoms of the cyanide group reflect the less strongly relative to the other atoms, the smaller the spacing of the planes, ${ }^{7}$ so that while qualitative agreement can be obtained using atomic numbers with the simple planes giving the spectrographic data, greater difficulty is found with the complicated planes of the Iaue photographs.

However, the various tests which have been applied to the Laue photographic data without resort to assumptions concerning the reflecting powers justify beyond much doubt the conclusions that the potassium atoms have Arrangement II; the zinc, cadmium, or mercury atoms Arrangement III; and the carbon and nitrogen atoms Arrangement V and VI with $1 / 2(u+v)$ equal to about 0.37 .

\section{Discussion of the Crystal Structures}

The arrangement of the zinc, cadmium, or mercury atoms is shown by the large cube of Fig. 4 in which $B G$ is equal to $d_{100}$. The arrangement

${ }^{7}$ Increasing departure from proportionality with atomic number with decreasing plane spacing in the case of $\mathrm{NaCl}$ is indicated by spectrometer measurements; W. I. Bragg, James, and Bosanquet, Phil. Mag., 41, 316 (1921). 
of the other atoms is shown by the small cubes in the lower part of the figure. The structure may be obtained by the alternation of the small cubes in the manner indicated by the lettering.

There is good evidence ${ }^{8}$ that in solution the ions $\mathrm{Zn}(\mathrm{CN})_{4}{ }^{=}, \mathrm{Cd}(\mathrm{CN})_{4}{ }^{=}$, and $\mathrm{Hg}(\mathrm{CN})_{4}=$ exist. One may well inquire whether there is evidence as to the existence of these ions in the solid state. In answer to this it may be pointed out that each cyanide group is situated differently with respect to some one zinc atom from what it is with respect to any other; and that each zinc atom has four cyanide groups surrounding it and identically related to it. No similar remarks apply to the potassium atoms. The atoms can accordingly be segregated into $\mathrm{Zn}(\mathrm{CN})_{+}$groups and $\mathrm{K}$ atoms without resorting to arbitrary selection.

On the other hand, it is remarkable that the structure of these complex cyanicles is of the same type as that previously found ${ }^{9}$ for spinel, $\mathrm{MgAl}_{2} \mathrm{O}_{4}$, and magnetite, $\mathrm{Fe}_{3} \mathrm{O}_{4}$. The cyanide group has taken the place of the oxygen atoms, the potassium the place of the aluminum or ferric iron, and the zinc the place of the magnesium or ferrous iron. The crystal structure evidence is, therefore, as much indicative of $\mathrm{FeO}_{4}$ groups and $\mathrm{MgO}_{4}$ groups as of $\mathrm{Zn}(\mathrm{CN})_{4}$ groups; but there seems to be little
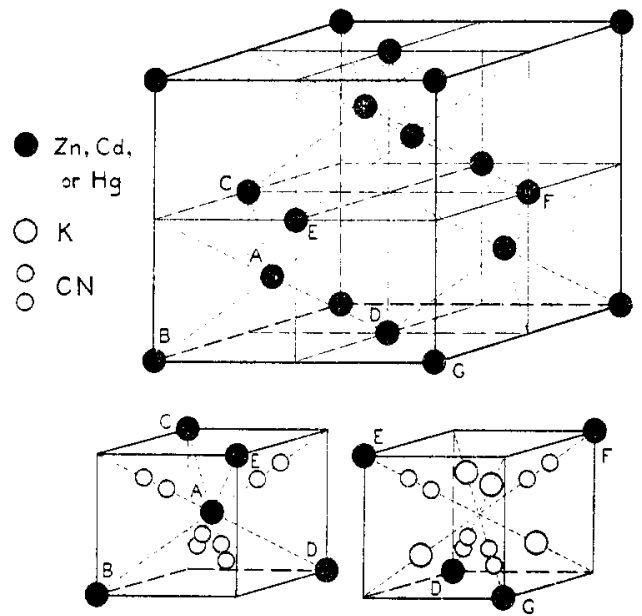

Fig. 4. - Arrangement of the atoms in $\mathrm{K}_{2} \mathrm{Zn}(\mathrm{CN})_{4}$, $\mathrm{K}_{2} \mathrm{Cd}(\mathrm{CN})_{4}$, or $\mathrm{K}_{2} \mathrm{Hg}(\mathrm{CN})_{4}$.

chemical evidence of the existence of $\mathrm{MgO}_{4}$ groups. If the likeness of crystal structure in the two classes of compounds is taken as evidence of correspondence of chemical affinities, ${ }^{10}$ either the $\mathrm{MgO}_{4}$ group must be accepted or the $\mathrm{Zn}(\mathrm{CN})_{4}$ group given up, in the crystalline state. It is plausible to assume that in the solid state the groups are themselves completely ionized into $\mathrm{Mg}^{++}$and $\mathrm{O}^{-}$ions and into $\mathrm{Zn}^{++}$and $\mathrm{CN}^{-}$ions.

The values of $d_{100}$ as well as the distance from the center of the cyanide group (whose position is probably more accurately known than the po-

8 Abegg, "Handbuch der Anorganische Chemie," Hirzel, Leipzig, 1905, II, 2, pp. $384,500,650$.

${ }^{9}$ W. H. Bragg, Phil. Mag., 30, 305 (1915). Nishikawa, Proc. Tokyo Math. Phys. Soc., 8, 199 (1915).

${ }^{10}$ The lack of definite cleavage in both series of crystals lends some support to this view. 
sitions of the individual carbon and nitrogen atoms) are summarized in Table VII. It will be noticed that the cadmium compound is not intermediate between the zinc and mercury compounds, but is built on a slightly larger scale than even the mercury compound. It will also be seen that, since the potassium atoms are situated relatively to the cyanide groups substantially as in potassium cyanide, ${ }^{6}$ the distances between their centers in each case may properly be compared. The agreement is surprisingly close; indeed, if the bivalent atoms of the complex cyanide are simply removed and replaced by twice as many potassium atoms in the proper position, the structure of potassium cyanide is almost quantitatively reproduced.

\begin{tabular}{cccc}
\multicolumn{5}{c}{ TABLE VII } \\
DistaNCES \\
Substance & $d_{100}$ & $\mathrm{~K}$ to $\mathrm{CN}$ & $\mathrm{Zn}_{\mathrm{n}}$ Cd, or $\mathrm{Hg}$ to $\mathrm{CN}$ \\
$\mathrm{K}_{2} \mathrm{Zn}(\mathrm{CN})_{4}$ & $12.54 \AA$. & $3.20 \AA$. & $2.61 \AA . \AA$ \\
$\mathrm{K}_{2} \mathrm{Cd}(\mathrm{CN})_{4}$ & 12.84 & 3.28 & 2.67 \\
$\mathrm{~K}_{2} \mathrm{Hg}(\mathrm{CN})_{4}$ & 12.76 & 3.25 & 2.65 \\
$\mathrm{KCN}$ & $1 / 2 \times 13.10$ & 3.28 & $\ldots$
\end{tabular}

VII. Summary

By means of spectral photographs and unsymmetrical Laue photographs, the structures of $\mathrm{K}_{2} \mathrm{Zn}(\mathrm{CN})_{4}, \mathrm{~K}_{2} \mathrm{Cd}(\mathrm{CN})_{4}$ and $\mathrm{K}_{2} \mathrm{Hg}(\mathrm{CN})_{4}$ have been determined. In the interpretation of the data the highly useful theory of space-groups has been employed, and it was not necessary to make any quantitative assumptions as to the "normal decline" of intensity. The results show that these compounds have the structure summarized at the end of Section $V$, represented by Fig. 4 , and discussed in Section VI. A remarkable fact is that these complex cyanides have the same structure as magnetite $\left(\mathrm{I}_{3} \mathrm{e}_{4} \mathrm{O}_{4}\right)$ and spinel $\left(\mathrm{MgAl}_{2} \mathrm{O}_{4}\right)$.

Pasadena, California

\section{NOTE}

A Simple Formula for the Calculation of the Specific Heats of Solids.The most important present day theories for the specific heat of solids agree in one respect, namely, that for a large class of solids, $C_{v}$ is the same function of the temperature, divided by a constant which is characteristic of the substance. In other words

$$
C_{v}=f\left(\frac{T}{\theta}\right)
$$

where $T$ is the absolute temperature, $\theta$ is the characteristic temperature for each substance and $f$ has the same form for all the substances under consideration. This is Equation $j$ in the treatment of specific heats given by Lewis and Gibson. ${ }^{1}$

' Lewis and Gibson, This Journal, 39, 2558 (1917). 